\title{
A Linear Time Algorithm for the Line Subgraph Problem in Halin Graphs
}

\author{
Dingjun Lou, Jun Liang and Guanpu Han \\ School of Data and Computer Science, Sun Yat-sen University, Guangzhou 510006, People's Republic of China
}

\begin{abstract}
Given a graph $\mathrm{G}=(\mathrm{V}, \mathrm{E})$ and a positive integer $\mathrm{p} \leq|\mathrm{E}|$. The Line Subgraph Problem is: Is there a subset $E^{\prime} \subseteq E$ such that $\left|E^{\prime}\right| \geq p$ and $H=(V, E)$ is a line graph. In this paper, we design a linear time algorithm to solve the line subgraph problem for Halin graphs. The algorithm is optimal.
\end{abstract}

keywords-halin graph; line graph; linear time algorithm

\section{INTRODUCTION AND TERMINOLOGY}

A Halin graph $\mathrm{H}$ is defined as follows: First, we embed a tree $\mathrm{T}$ in the plane such that each inner vertex of $\mathrm{T}$ has degree at least 3 , then we draw a cycle $\mathrm{C}$ through all leaves of $\mathrm{T}$ to form a planar graph. Then $\mathrm{H}=\mathrm{T} \cup \mathrm{C}$ is called a Halin graph, where $\mathrm{T}$ is called the characteristic tree of $\mathrm{H}$ and $\mathrm{C}$ is called the accompanying cycle of $\mathrm{H}$. An example of a Halin graph is shown in Figure 1.

The simplest Halin graphs are wheels. Suppose a Halin graph $\mathrm{H}=\mathrm{T} \cup \mathrm{C}$ is not a wheel. If $\mathrm{w}$ is an inner vertex of $\mathrm{T}$ such that all neighbors $\mathrm{v}_{1}, \mathrm{v}_{2}, \ldots, \mathrm{v}_{\mathrm{k}}$ of $\mathrm{w}$ except one neighbor are leaves of $\mathrm{T}$, then the induced subgraph $\mathrm{H}\left[\left\{\mathrm{w} \cup\left\{\mathrm{v}_{1}, \mathrm{v}_{2}, \ldots, \mathrm{v}_{\mathrm{k}}\right\}\right]\right.$ is called a fan of $\mathrm{H}$ and $\mathrm{w}$ is called the center of the fan (See Figure 1). We define a fan with $\mathrm{k}$ leaves of $\mathrm{T}$ to be a $\mathrm{k}$-fan. If an inner vertex $w^{\prime}$ of $T$ is not the center of a fan but $w^{\prime}$ is adjacent to $\mathrm{k}$ consecutive leaves $\mathrm{v}_{1}, \mathrm{v}_{2}, \ldots, \mathrm{v}_{\mathrm{k}}$ of $\mathrm{T}$ on $\mathrm{C}$, then $\mathrm{H}\left[\left\{\mathrm{w}^{\prime}\right\} \cup\left\{\mathrm{v}_{1}, \mathrm{v}_{2}, \ldots, \mathrm{v}_{\mathrm{k}}\right\}\right]$ is called a pseudo $\mathrm{k}$-fan, in particular, if $\mathrm{k}=1$, then the pseudo $\mathrm{k}$-fan is called a pseudo single fan.

For all terminology and notation not defined in this paper, the reader is referred to [1].

Halin graphs were introduced by German mathematician Halin [3] as minimally 3-connected planar graphs. It can be used as a model of a network with minimum cost and fault tolerance. Halin graphs have many good properties. For example, many NP-complete or NP-hard problems restricted on Halin graphs can be solved in polynomial time or even in linear time.

The TSP problem for general graphs is an NP-hard problem. However, Cornuejols, Naddef and Pulleyblank [2] give a linear time algorithm to solve the TSP for a weighted Halin graph. Lou [5] proves that each Halin graph is Hamiltonian connected, that is, for each pair of vertices $u$ and $\mathrm{v}$ in a Halin graph $\mathrm{H}$, there is a Hamiltonian path from $\mathrm{u}$ to $\mathrm{v}$ in $\mathrm{H}$. Then $\mathrm{Li}$, Lou and $\mathrm{Lu}$ [4] design a linear time algorithm

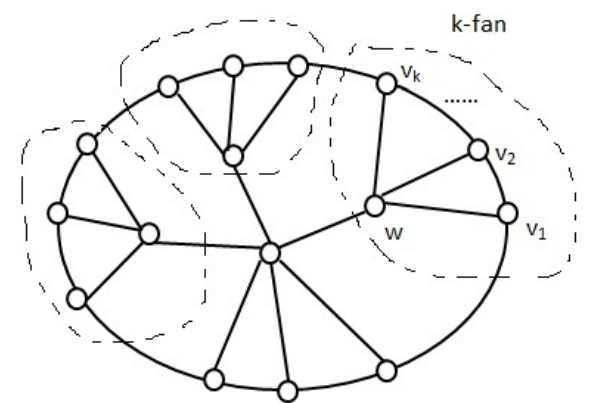

FIGURE I. AN EXAMPLE FOR A HALIN GRAPH

to find a Hamiltonian path with minimum weight between each pair of vertices in a weighted Halin graph.

Phillips, Punnen and Kabadi [7] design a linear time algorithm to solve the Bottleneck TSP in a weighted Halin graph. Lou and Liu [6] give a linear time algorithm to solve the cubic subgraph problem for Halin graphs, which is to find a cubic subgraph with maximum number of edges in a Halin graph. The problems mentioned above for general graphs are all NP-complete or NP-hard.

In this paper, we give a linear time algorithm to solve the line subgraph problem for Halin graphs. The line subgraph problem is: Given a graph $\mathrm{G}=(\mathrm{V}, \mathrm{E})$ and a positive integer $\mathrm{p} \leq|\mathrm{E}|$. Is there a subset $\mathrm{E}^{\prime} \subseteq \mathrm{E}$ such that $\left|\mathrm{E}^{\prime}\right| \geq \mathrm{p}$ and $\mathrm{H}=\left(\mathrm{V}, \mathrm{E}^{\prime}\right)$ is a line graph.

The line subgraph problem for general graphs is also NPcomplete. We restrict the problem on Halin graphs and design a linear time algorithm to solve the problem, the algorithm is optimal.

The line graph $\mathrm{H}$ of a graph $\mathrm{G}=(\mathrm{V}, \mathrm{E})$ is such a graph that, for each edge e $\in E(G), H$ has a vertex ve corresponding to e, two vertices ve and vf of $\mathrm{H}$ are adjacent if and only if their corresponding edges $e$ and $f$ are adjacent in $G$ (i.e. e and $f$ have a common end vertex in $G$ ). Such a graph $G$ is called the base graph of $\mathrm{H}$.

\section{ANALyzing The Structure Of A Halin GRAPH}

In the following, we present a property of the line graphs which is a necessary condition for a graph to be a line graph. 


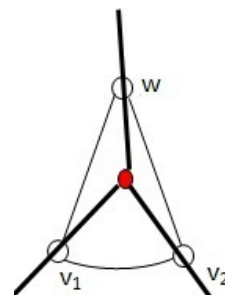

(a)

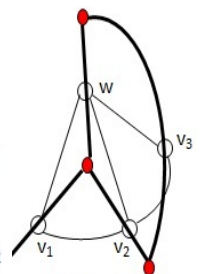

(b)

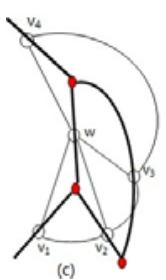

FIGURE II. THE STRUCTURE W IS A FAN

Lemma 2.1 A line graph is a claw-free graph.

Lemma 2.1 is a well-known property of the line graphs. A claw of a graph $\mathrm{G}$ is an induced subgraph isomorphic to $\mathrm{K} 1,3$.

Now we analyze the structure of a Halin graph $\mathrm{H}=\mathrm{T} \cup \mathrm{C}$ to find a base graph of a structure $\mathrm{W}$ of $\mathrm{H}$, where the base graph is drawn with thick lines, and $\mathrm{W}$ is drawn with thin lines.

Case (1): The structure $\mathrm{W}$ is a fan.

Case (1.1): If $\mathrm{W}$ is a 2-fan $\mathrm{H}[\{\mathrm{w}\} \cup\{\mathrm{v} 1, \mathrm{v} 2\}]$, then its base graph exists (See Figure 2(a)). So 2-fan is a line subgraph.

Case (1.2): If $W$ is a 3 -fan $H[\{w\} \cup\{v 1, v 2, v 3\}]$, then its base graph exists (See Figure 2(b)). So 3-fan is a line subgraph.

Case (1.3): If $\mathrm{W}$ is a 4-fan $\mathrm{H}[\{\mathrm{w}\} \cup\{\mathrm{v} 1, \mathrm{v} 2, \mathrm{v} 3, \mathrm{v} 4\}]$, then its base graph exists (See Figure 2(c)). So 4-fan is a line subgraph.

Case (1.4): If $\mathrm{W}$ is a $\mathrm{k}-\mathrm{fan}(\mathrm{k} \geq 5) \mathrm{H}[\{\mathrm{w}\} \cup\{\mathrm{v} 1, \mathrm{v} 2, \ldots, \mathrm{vk}\}]$, then $\mathrm{H}[\{\mathrm{w}\} \cup\{\mathrm{v} 1, \mathrm{v} 3, \mathrm{v} 5\}]$ is a claw, so $\mathrm{W}$ is not a line subgraph, and we shall delete the edges wv5, wv6, ..., wvk to find a line subgraph.

Case (2): The center of a fan is adjacent to an inner vertex of T.

Case (2.1): If the fan $\mathrm{W}$ is a 2-fan, then the center of $\mathrm{W}$ can be adjacent to an inner vertex of $\mathrm{T}$, the base graph exists (See Figure 3).

Case (2.2): If the fan $\mathrm{W}$ is a $\mathrm{k}-\mathrm{fan}(\mathrm{k} \geq 3), \mathrm{W}=\mathrm{H}[\{\mathrm{w}\} \cup\{\mathrm{v} 1$, $\mathrm{v} 2, \ldots, \mathrm{vk}\}]$, and if $\mathrm{w}$ is adjacent to an inner vertex $\mathrm{x}$ of $\mathrm{T}$, then $\mathrm{H}[\{\mathrm{w}\} \cup\{\mathrm{x}, \mathrm{v} 1, \mathrm{v} 3\}]$ is a claw. So, to find a line subgraph, the center of a $k$-fan $(k \geq 3)$ cannot be adjacent to any inner vertex of T.

Case (3): The leaves of $\mathrm{T}$ in a fan $\mathrm{W}$ are adjacent to other vertices on $\mathrm{C}$.

Case (3.1): If $\mathrm{W}$ is a 2 -fan, and $\mathrm{W}=\mathrm{H}[\{\mathrm{w}\} \cup\{\mathrm{v} 1, \mathrm{v} 2\}]$, then $\mathrm{v} 1$ and $\mathrm{v} 2$ can be adjacent to the neighbors on $\mathrm{C}$, the base graph exists (See Figure 4(a)). In fact, v1 (v2) can be connected by a path on C to v5 (v3) in the line subgraph.

$\mathrm{v} 4\}$ ], then $\mathrm{v} 1$ and $\mathrm{v} 4$ can be adjacent to the neighbors v5 and $\mathrm{v} 7$ respectively on $\mathrm{C}$ (See Figure 4(c)). In fact, v1 (v4) can be connected by a path on $\mathrm{C}$ to $\mathrm{v} 5$ (v7) in the line subgraph. fans.

Case (4): An inner vertex $\mathrm{w}$ of $\mathrm{T}$ is contained in pseudo

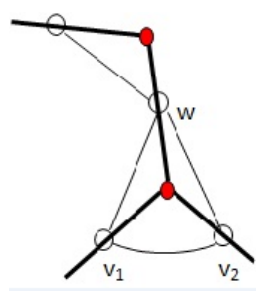

FIGURE III. THE CENTER OF A 2-FAN IS ADJACENT TO AN INNER VERTEX OF T

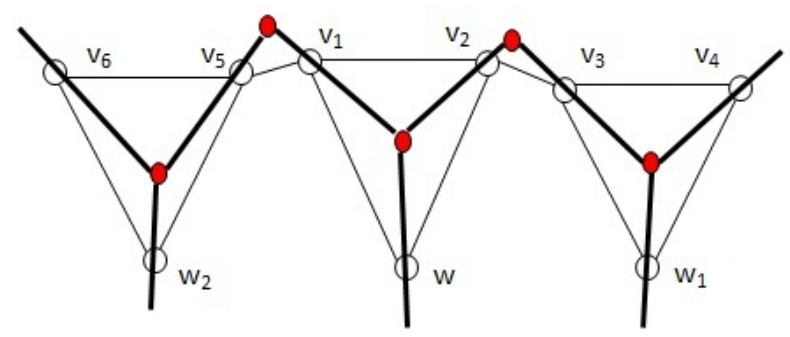

(a)

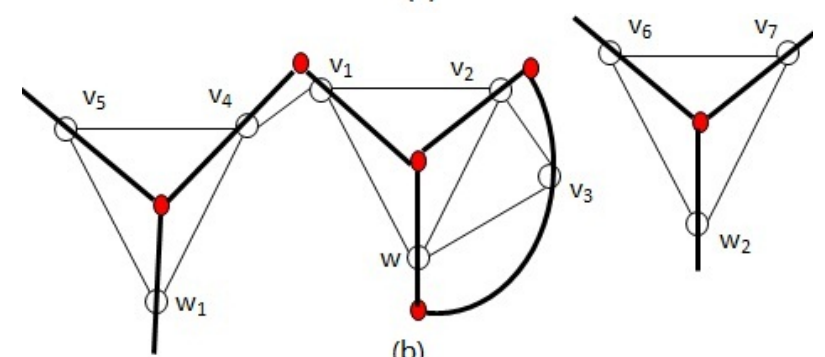

(b)

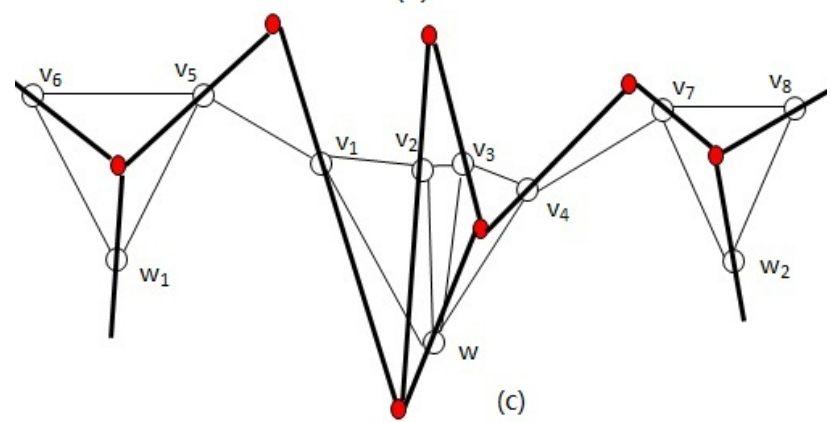

FIGURE IV. THE LEAVES OF T IN A FAN W ARE ADJACENT TO OTHER VERTICES ON C

Case (3.2): If $\mathrm{W}$ is a 3-fan, and $\mathrm{W}=\mathrm{H}[\{\mathrm{w}\} \cup\{\mathrm{v} 1, \mathrm{v} 2, \mathrm{v} 3\}]$, then $\mathrm{v} 1$ can be adjacent to a neighbor $\mathrm{v} 4$ on $\mathrm{C}$, but $\mathrm{v} 3$ cannot (See Figure 4(b)). So, to find a line subgraph with maximum number of edges, we must delete one edge wv3, then treat the fan $\mathrm{W}-\mathrm{wv} 3$ as a 2 -fan. Then $\mathrm{w}$ can be adjacent to an inner vertex of $\mathrm{T}$ and $\mathrm{v} 3$ can be adjacent to the neighbor $\mathrm{v} 6$ on $\mathrm{C}$.

Case (3.3): If $\mathrm{W}$ is a 4-fan, and $\mathrm{W}=\mathrm{H}[\{\mathrm{w}\} \cup\{\mathrm{v} 1, \mathrm{v} 2, \mathrm{v} 3$,

Case (4.1): If the inner vertex $\mathrm{w}$ of $\mathrm{T}$ is contained in a pseudo single fan $\mathrm{W}=\mathrm{H}[\{\mathrm{w}\} \cup\{\mathrm{v} 1\}]$, then $\mathrm{H}[\{\mathrm{v} 1\} \cup \mathrm{NH}(\mathrm{v} 1)]$ is a claw. So the edge wv1 will be deleted to find a line subgraph.

Case (4.2): If the inner vertex $\mathrm{w}$ of $\mathrm{T}$ is contained in one pseudo 2-fan $\mathrm{W}=\mathrm{H}[\{\mathrm{w}\} \cup\{\mathrm{v} 1, \mathrm{v} 2\}]$, similar to $\operatorname{Cases}(1.1)$, 
(2.1) and (3.1), W can be kept and $\mathrm{w}$ can be adjacent to an inner vertex of $\mathrm{T}$, and $\mathrm{v} 1(\mathrm{v} 2)$ can be adjacent to its neighbors on $\mathrm{C}$.

Case (4.3): If the inner vertex $\mathrm{w}$ of $\mathrm{T}$ is contained in one pseudo 3 -fan $\mathrm{W}=\mathrm{H}[\{\mathrm{w}\} \cup\{\mathrm{v} 1, \mathrm{v} 2, \mathrm{v} 3\}]$, then the edge wv3 will be deleted, w can be adjacent to an inner vertex of $\mathrm{T}$ and $\mathrm{v} 1(\mathrm{v} 3)$ can be adjacent to its neighbors on $\mathrm{C}$.

Case (4.4): If the inner vertex $\mathrm{w}$ of $\mathrm{T}$ is contained in one pseudo 4-fan $\mathrm{W}=\mathrm{H}[\{\mathrm{w}\} \cup\{\mathrm{v} 1, \mathrm{v} 2, \mathrm{v} 3, \mathrm{v} 4\}]$, similar to Cases(1.3), (2.2) and (3.3), W can be kept, w cannot be adjacent to any inner vertex of $\mathrm{T}$, and $\mathrm{v} 1(\mathrm{v} 4)$ can be adjacent to its neighbors on $\mathrm{C}$.

Case (4.5): If the inner vertex $\mathrm{w}$ of $\mathrm{T}$ is contained in one pseudo $\mathrm{k}$-fan $\mathrm{W}=\mathrm{H}[\{\mathrm{w}\} \cup\{\mathrm{v} 1, \mathrm{v} 2, \ldots, \mathrm{vk}\}]$ for $\mathrm{k} \geq 5$, similar to Cases(1.4), (2.2) and (3.3), W is not a line subgraph, we shall delete the edges wv5, wv6, ..., wvk, w cannot be adjacent to any inner vertex of $\mathrm{T}$ and $\mathrm{vl}(\mathrm{vk})$ can be adjacent to its neighbors on $\mathrm{C}$.

Case (4.6): If the inner vertex $\mathrm{w}$ of $\mathrm{T}$ is contained in at least two pseudo fans $\mathrm{W} 1=\mathrm{H}[\{\mathrm{w}\} \cup\{\mathrm{v} 1, \mathrm{v} 2, \ldots, \mathrm{vr}\}]$, $\mathrm{W} 2=\mathrm{H}[\{\mathrm{w}\} \cup\{\mathrm{u} 1, \mathrm{u} 2, \ldots, \mathrm{us}\}](\mathrm{r} \geq 2$ and $\mathrm{s} \geq 2)$ and $\mathrm{W} 3, \mathrm{~W} 4, \ldots$, $\mathrm{Wt}$, then delete the edges wv3, wv4, ..., wvr, wu3, wu4, ..., wus and all edges from $\mathrm{w}$ to the leaves of $\mathrm{T}$ in $\mathrm{W} 3, \mathrm{~W} 4, \ldots, \mathrm{Wt}$, and $\mathrm{w}$ cannot be adjacent to any inner vertex of $\mathrm{T}$ and the edges on $\mathrm{C}$ incident with (or contained in) $\mathrm{W} 1, \mathrm{~W} 2, \ldots, \mathrm{Wt}$ remain. The remaining graph is a line subgraph (See Figure 5).

Case (5): The structure of inner vertices of $\mathrm{T}$.

If $w$ is an inner vertex of $T$ such that $w$ is not the center of a fan or a pseudo fan, $\mathrm{dT}(\mathrm{w}) \geq 3$, and the vertices in $\mathrm{NH}(\mathrm{w})$ are inner vertices of $\mathrm{T}$, then $\mathrm{H}[\{\mathrm{w}\} \cup \mathrm{NH}(\mathrm{w})]$ forms a claw. So the inner vertices of $\mathrm{T}$ form a line subgraph if and only if they form a forest with each tree to be a path by deleting some edges of $T$.

To obtain a line subgraph with maximum number of edges in T-S ( $\mathrm{S}$ is the set of all leaves of T), the inner vertices of T form a spanning subgraph T' of T-S, which is a set of paths. T' satisfies the following properties: The vertices $\mathrm{x} 1, \mathrm{x} 2, \ldots, \mathrm{xr}$ of degree less than 2 in $\mathrm{T}^{\prime}$ are adjacent (in T) to vertices $\mathrm{y} 1$, $\mathrm{y} 2, \ldots$, ys of degree 2 (in $\mathrm{T}^{\prime}$ ) but $\mathrm{x} 1, \mathrm{x} 2, \ldots, \mathrm{xr}$ are not adjacent to $\mathrm{y} 1, \mathrm{y} 2, \ldots, \mathrm{ys}$ in $\mathrm{T}$; ; or $\mathrm{x} 1, \mathrm{x} 2, \ldots, \mathrm{xr}$ are adjacent (in T) to vertices $\mathrm{w} 1, \mathrm{w} 2, \ldots, \mathrm{wt}$, where $\mathrm{w} 1, \mathrm{w} 2, \ldots, \mathrm{wt}$ are centers of k-fans or pseudo k-fans, but by Cases (2.2), (3.2) and (4), w1, w2, .., wt are not adjacent to $\mathrm{x} 1, \mathrm{x} 2, \ldots, \mathrm{xr}$ in T'; or xi belongs to $\{w 1, w 2, \ldots, w t\}$ for some i's, and by Cases (2.2), (3.2) and (4), xi has degree less than 2 in T'.

To see that $T$ ' has maximum number of edges, suppose another spanning line subgraph $T$ " has maximum number of edges, but xi $(1 \leq i \leq r)$ has degree $\mathrm{dT}^{\prime}(\mathrm{xi})+1$ in T", then xiyj belongs to $E\left(T^{\prime \prime}\right)$, but then yj has degree at least 3, so at least one edge incident with yj (in T' ) must be deleted in T"; or xiwj

belongs to E(T"), but by Cases (2.2), (3.2) and (4), wj cannot have degree more than 1 , so the edge incident with wj in T' must be deleted in T'; or xi belongs to $\{\mathrm{w} 1, \mathrm{w} 2, \ldots$, wt \}, by Cases (2.2), (3.2) and (4), xi cannot have degree more than its original degree in T', otherwise the above two cases happen. We can obtain T" from T' by a series of operations as above three cases. We can see that T" will not have more edges than T'.

\section{III.ALGORITHM TO FIND A MAXIMUM LINE SUBGRAPH}

For any given Halin graph $\mathrm{H}=\mathrm{T} \cup \mathrm{C}$, we use the following algorithm to find a maximum line subgraph for $\mathrm{H}$.

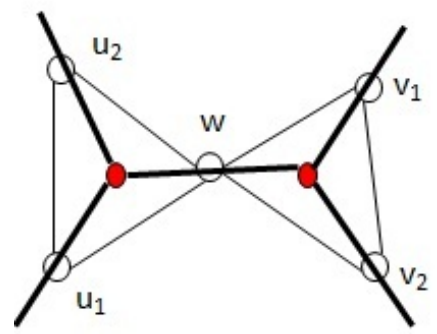

FIGURE V. THE INNER VERTEX W OF T IS CONTAINED IN AT LEAST TWO PSEUDO K-FANS

\section{Algorithm:}

1. We do postorder traversal of $T$ and choose an inner vertex $r$ of $T$ as the root such that $r$ is not the center of any fan if there is such a vertex; otherwise $r$ is any inner vertex of $T$.

2. When we visit an inner vertex $\mathrm{w}$ of $\mathrm{T}$ in the postorder traversal,

(2.1) If $\mathrm{w}$ is the center of a wheel $\mathrm{W}=\mathrm{H}[\{\mathrm{w}\} \cup\{\mathrm{v} 1, \mathrm{v} 2, \ldots$, $\mathrm{vk}\}]=\mathrm{H}$, and if $\mathrm{k} \geq 4$, then delete $\mathrm{wv} 5$, wv6, .., wvk from $\mathrm{H}$; if $\mathrm{k}=3$, then delete $\mathrm{w} v 3$. The resulting graph is a maximum line subgraph.

(2.2) If $\mathrm{w}$ is the center of a $\mathrm{k}-\mathrm{fan} \mathrm{W}=\mathrm{H}[\{\mathrm{w}\} \cup\{\mathrm{v} 1, \mathrm{v} 2, \ldots$, $\mathrm{vk}\}$,

(2.2.1) If $\mathrm{k}=2$, keep the edge joining $\mathrm{w}$ to another inner vertex in $\mathrm{T}$;

(2.2.2) If $\mathrm{k}=3$, delete the edge wv3, and keep the edge joining $\mathrm{w}$ to another inner vertex in $\mathrm{T}$;

(2.2.3) If $\mathrm{k}=4$, delete the edge joining $\mathrm{w}$ to another inner vertex of $\mathrm{T}$;

(2.2.4) If $k \geq 5$, delete the edges wv5, wv6, .., wvk and the edge joining $\mathrm{w}$ to another inner vertex of $\mathrm{T}$;

(2.3) If $\mathrm{w}$ is contained in a pseudo $\mathrm{k}$-fan $\mathrm{W} 1=\mathrm{H}[\{\mathrm{w}\} \cup$ $\{\mathrm{v} 1, \mathrm{v} 2, \ldots, \mathrm{vk}\}$,

(2.3.1) While $\mathrm{w}$ is contained in a pseudo single fan $\mathrm{W} 1=\mathrm{H}$ $[\{\mathrm{w}\} \cup\{\mathrm{v} 1\}]$, do deleting the edge wv1;

\section{(2.3.2) If $\mathrm{w}$ is contained in one pseudo $\mathrm{k}$-fan $\mathrm{W} 1$, then}

(2.3.2.1) If $\mathrm{k}=2$, keep only one edge which joins $\mathrm{w}$ to another inner vertex as its child in $\mathrm{T}$; if there is not such a child, then keep the edge to its father; Then delete the other edges joining $\mathrm{w}$ to other inner vertices of $\mathrm{T}$;

(2.3.2.2) If $\mathrm{k}=3$, delete wv3, then do the same work as (2.3.2.1); 
(2.3.2.3) If $\mathrm{k}=4$, delete the edges joining $\mathrm{w}$ to other inner vertices of $\mathrm{T}$;

(2.3.2.4) If $\mathrm{k} \geq 5$, delete the edges wv5, wv6, .., wvk and do the same work as (2.3.2.3).

(2.3.3) If $\mathrm{w}$ is contained in at least two pseudo fans $\mathrm{W} 1=\mathrm{H}[\{\mathrm{w}\} \cup\{\mathrm{v} 1, \mathrm{v} 2, \ldots, \mathrm{vr}\}], \mathrm{W} 2=\mathrm{H}[\{\mathrm{w}\} \cup\{\mathrm{u} 1, \mathrm{u} 2, \ldots, \mathrm{us}\}]$ and $\mathrm{W} 3, \mathrm{~W} 4, \ldots, \mathrm{Wt}$, and $\mathrm{r}, \mathrm{s} \geq 2$, then delete $\mathrm{wv} 3$, wv $4, \ldots$, wvr; wu3, wu4, ..., wus; and all edges joining $\mathrm{w}$ to the leaves of $\mathrm{T}$ in $\mathrm{W} 3, \mathrm{~W} 4, \ldots, \mathrm{Wt}$. Then delete the edges joining $\mathrm{w}$ to other inner vertices of $\mathrm{T}$.

(2.4) If $\mathrm{w}$ is only adjacent to inner vertices of $\mathrm{T}$, then two edges to its children are kept; if $\mathrm{w}$ is adjacent to only one child, then we keep one edge to its child and one edge to its father. Then we delete the other edges incident with w. If there is only one edge incident with w, we keep it.

3 . When we have visited the root $r$, all vertices have been processed as in Step 2, and then the resulting graph is a maximum line subgraph.

By the analysis of the structure of a Halin graph $\mathrm{H}$ in Section 2, we can see that our algorithm is correct. Since our algorithm only do once postorder traversal of $\mathrm{T}$, the worst time complexity of the algorithm is $\mathrm{O}(\mathrm{n})$, where $\mathrm{n}$ is the number of vertices in $\mathrm{H}$ (we shall also notice that $|\mathrm{E}(\mathrm{H})|=|\mathrm{V}(\mathrm{H})|=\mathrm{O}(\mathrm{n})$ ).

\section{REFERENCES}

[1] J.A. Bondy and U.S.R. Murty, Graph theory withapplications, Macmillan Press, London (1976).

[2] G.Cornuejols, D.Naddef and W.Pulleyblank, Halin graphs and the traveling salesman problem, Mathematical Programming 26(1983), 287-294.

[3] R.Halin, Studies on minimally n-connected graphs,Combinatorial Mathematics and its Applications,Academic Press, London(1971), 129136.

[4] Y.Li, Dingjun Lou, Y.Lu, Algorithms for the optimal Hamiltonian path in Halin graphs, Ars Combinatoria 87(2008), 235-255.

[5] Dingjun Lou, Hamiltonian paths in Halin graphs,Mathematica Applicata(Chinese) 8:2(1995), 158-160.

[6] Dingjun Lou and Junfu Liu, A linear time algorithm for cubic subgraph of Halin graphs, Proceedings of the 2nd International Conference on Computer Science and Electronics Engineering (ICCSEE 2013) (2013), 386-388.

[7] J.M.Phillips, A.P.Punnen and S.N.Kabadi, A linear time algorithm for the bottleneck traveling salesman problem on a Halin graph, Information Processing Letters, 67(1998), 105-110. 